\title{
Immersive Mixed Media Augmented Reality Applications and Technology
}

\author{
Volker Kuchelmeister, Jeffrey Shaw, Matthew McGinity, Dennis Del Favero, \\ Ardrian Hardjono \\ UNSW University of New South Wales Sydney, iCinema Centre for Interactive Cinema \\ Research.kuchel@unsw.edu.au
}

\begin{abstract}
In this paper I will outline an augmented reality system based on high resolution omni-directional motion image capture, an immersive interactive projection environment, the iDome and multi-layered narrative strategies and applications for educational and entertainment use. This system differs in two ways from other AR frameworks. It operates in non real-time, by employing $360^{\circ}$ video recording instead of a live camera feed and it uses immersive projection technology, a $180^{\circ}$ dome surface as a display environment. Not being constrained by the requirements of a real-time system, the proposed framework can use high-resolution imagery and immersive display technology to deliver an engaging and true to life experience for the user.
\end{abstract}

Keywords: Interactive Environment, Omni-directional capture, Immersive Projection Environment, Multi-layered Narrative

\section{Technical Framework}

\section{1 iDome Immersive Environment}

The iDome [8] is a proprietary hardware/software platform that offers a cost-effective and compact immersive visualisation environment for panoramic and spherical representations, video and/or computer generated. It utilizes a three meter fiberglass dome as the surface for $180^{\circ}$ projection made possible by a High Definition projector and a spherical mirror as reflection surface [1,2,3]. Its flexibility, immersive qualities and cost-effectiveness does make it an ideal platform for applications such as data visualisation in research, Virtual Reality in training and research and interactive museum exhibits. 

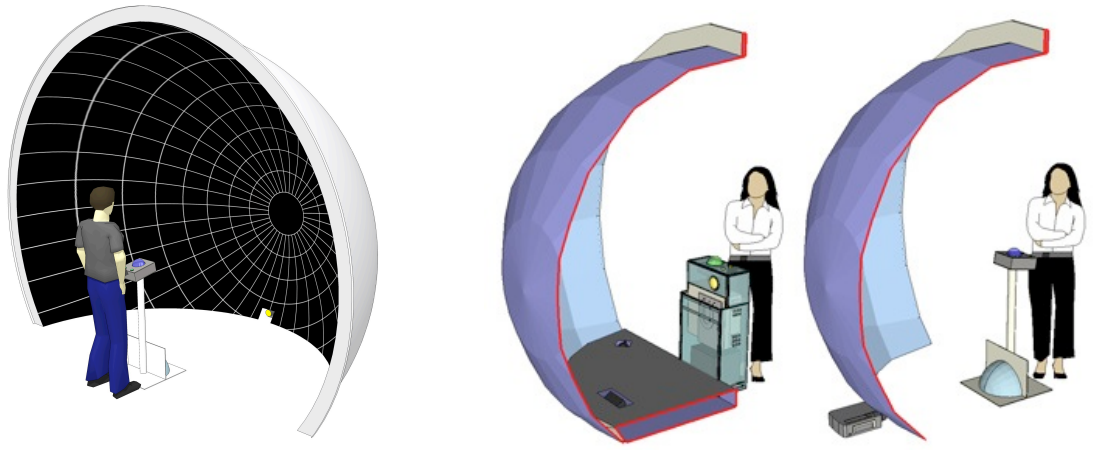

Figure 1. iDome model (1), two projection methods, fisheye projection lens (m) and spherical mirror approach (r).

Two approaches to project into and fill the hemisphere with imagery are utilized. A custom $180^{\circ}$ fisheye projection lens located in the center of the dome or a spherical mirror as reflection surface [2] for a projector located behind the dome (Fig. 1).

A custom 3D software engine takes a high-resolution spherical/equirectangular image or video stream and uses it as a texture map projected on the inside of a sphere. The geometric correction for the mirror in relation to the dome surface is undertaken through a distortion mesh in the render pipeline. The video texture is translated inside the sphere according to the point of view of the user, which simulates "looking around" in the scene. Spherical/equirectangular images or movies are projected inside the dome with a coverage of 180 degree. A track ball or other type of interface allows the user to rotate the projection freely while the movie is still running. Together with the image, a multi-channel directional sound field is rotated accordingly. A simple vector based panning algorithm is used to distribute the multi-channel sound dependent of the user's principle point of view across five speakers positioned around the viewer.

The immersive quality of the iDome system is derived from its $180^{\circ}$ wide field of view, covering the peripheral vision of a viewer standing in front of it completely. The ideal location or "sweet spot" for immersion and correct geometrical appearance is in the center of the hemisphere, but experience shows that up to 6 people can view a scene in the iDome comfortable.

\subsection{Omni-directional Capture Systems}

\subsubsection{Spherecam}

The Spherecam [7] is a custom integrated digital video camera cluster with panoramic and/or spherical recording capability. The system is comprised of an omni-directional dioptric-camera head, mounting plates, an Ambisonic microphone and recording racks. Its compact $\mathrm{CCD}$ color cameras ${ }^{1}$ allow the capturing of high resolution images

${ }^{1} 12 \times$ Imperx IPX-2M30G, 1600x1200 pixel resolution, $11.84 \times 8.88 \mathrm{~mm}$ active image area, digital $8 / 10 / 12$ bits video output, GigE interface, up to $33 \mathrm{fps}, 1 / 40000$ to $1 / 15 \mathrm{sec}$ shutter speed, C-mount 
in full video/cinematic frame rates. Image sensing, capture and recording are all in the digital domain, ensuring high image quality.
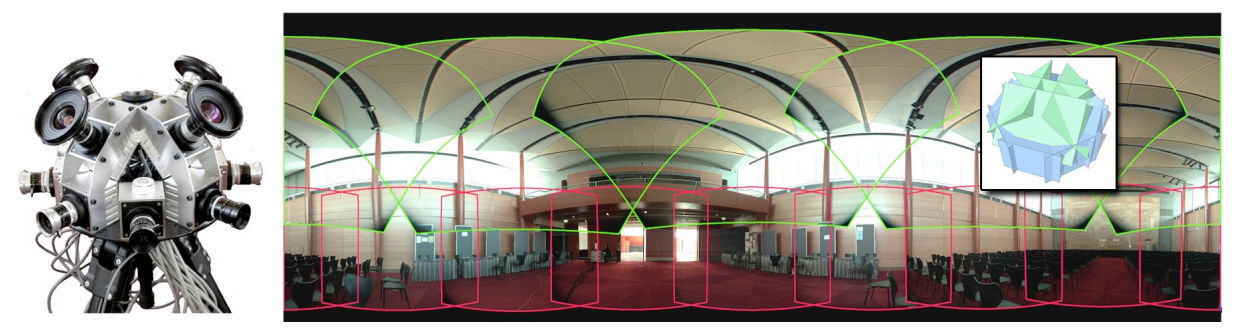

Figure 2. Spherecam in spherical $8 \times 4$ (8 cameras horizontal, $445^{\circ}$ vertical) configuration (1) and video still in equirectangular projection (cropped). The inset shows the coverage and overlap of individual camera image planes.

Depending on the desired vertical field of view, image resolution, distance to the near plane and the intended playback context, a specific camera cluster arrangement is chosen. This includes a panoramic mode (12 cameras in a ring cluster, $12 \times 0)$, a semi spherical mode ( 8 cameras in a ring and 4 pointing up, $8 \times 4$ ) and full spherical modes, with either 3 cameras and $180^{\circ}$ fisheye lenses or 8 cameras in a ring and one camera with fisheye lens pointing up. A variety of lenses are used to suite these configurations.

\subsubsection{Ladybug Camera}

The Ladybug 2 is a commercially available portable and compact medium-resolution spherical video camera from Point Grey Research. It enables rapid prototyping to be executed in the field or in situations where only a small portable device would be serviceable. Recently the next generation Ladybug 3 became available, increasing the resolution to 5600 pixel horizontal, but with a limit of 15 frames per second [9].
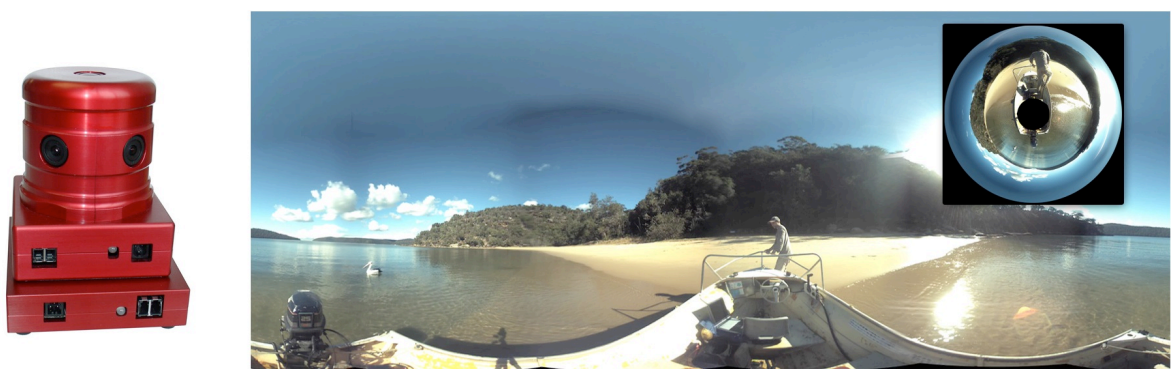

Figure 3. Pointgrey Research Ladybug 2 camera (1), Video still in equirectangular projection (cropped) and polar projection (inset) (r)

The camera has a tightly packed cluster of 6 CCD sensors with wide angle lenses and a slight overlap between the images. In post production the individual frames are color and geometrically corrected and stitched to a high resolution equirectangular image [4]. 


\begin{tabular}{|l|l|l|l|}
\hline Display system & Field of view & Resolution [pixel] & Frame rate \\
\hline iDome & $180^{\circ} \times 112^{\circ}$ & $1920 \times 1080$ & 60 \\
\hline Capture system & Field of view & Resolution [pixel] & Frame rate \\
\hline Spherecam $8 \times 1$ & $360^{\circ} \times 120^{\circ}$ & $5300 \times 1600$ & 30 uncompressed \\
\hline Spehecam $8 \times 4$ & $360^{\circ} \times 104^{\circ}$ & $5300 \times 1500$ & 30 uncompressed \\
\hline Ladybug 2 & $360^{\circ} \times 120^{\circ}$ & $3500 \times 1160$ & 30 compressed \\
\hline Ladybug 3 & $360^{\circ} \times 120^{\circ}$ & $5600 \times 1860$ & 15 compressed \\
\hline
\end{tabular}

Table 1. Display and capture systems in comparison

\section{APPLICATIONS}

\subsection{Conversation@ the Studio, 2005}

\subsubsection{Introduction}

Conversations@the Studio [6] (Fig. 4) presents an innovative mixed-reality narrative model of the Powerhouse Museum, Sydney, Decorative Arts collection within a uniquely designed Intelligent Interactive Information Environment (IIIE). It takes the natural navigation of a real world situation - a contemporary Glass Studio - as its point of departure, using this as a framework for organizing a set of narrative formations that further elaborate the thematics of the collection. By visualising a 360 degree global video recording, made on location at the Glass Studio, it provides the telepresent experience of an actual visit to the Studio, giving full interactive freedom to the viewer's gaze. Multilayered narrative extensions are embedded within the global video recording. This is articulated by multiple audio streams, and by conjunctions between the global scene and augmented close-up sequences that detail the glass handling process.

\subsubsection{Installation}

The highly successful installation at the Powerhouse takes the form of a custom designed three-meter diameter hemispherical fiberglass projection screen that is mounted vertically so that viewers look straight ahead into its immersive concave projection space. A high-resolution projector fitted with a unique 180 degree fisheye lens is mounted in front of the screen, and the projection is augmented by a 5 channel surround sound system. The specifically developed user interface is an over-sized illuminated track ball that allows navigation of the global video recording, it changes color in dynamic relation to the content displayed on the screen. This latter functionality is adapted to signaling the availability of close-up sequences within the global scene. Additional controls activate the voice-over commentary and offer a fast forward function.

The IIIE forms the central digital focus of the Museum's new permanent gallery showcasing artifacts from its Decorative Arts and Design collection. The IIIE utilizes an integrated design strategy that is driven by the specific nature of the Museum's decorative arts content, enriching the content by offering multiple layers of multimedia production that augment methods of documentation using 360 degree 
global recording. Innovative visualisation and interface strategies incorporate these recordings in an environment where the viewer can encounter and explore the content in a dynamic manner.
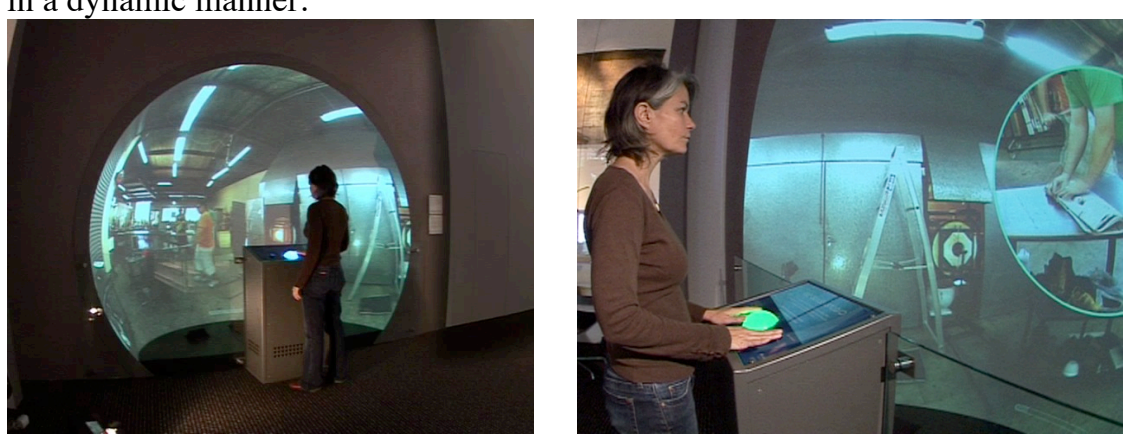

Figure 4. Conversations@the Studio Installation at the Powerhouse Museum Sydney, 2005.

\subsubsection{Benchmark}

Conversations@the Studio sets a new museological multi-media benchmark. Its interactive and immersive frameworks allow the viewer to experience themselves present in a distant space where artists are engaged in their daily creative craft making activities. They can move their gaze freely anywhere in the live performance space whose unedited real time narration converges with the real time engagement of the viewer. This space-time continuum between the local (Powerhouse Museum) and remote (glass studio) location enables the viewer to achieve an immediacy of experience that has hitherto not been achievable in multi-media exhibits. This is reinforced by the dynamic surround sound reproduction of the glass studio environment that is sensitive to the virtual position of the viewer's gaze. A key feature of Conversations@the studio is the dynamic tracking of the viewer's gaze in both space and time that visually magnifies specific events of interest (Fig. 5, 6). This polychronic cinematic operability gives the viewer a multi-visual faculty that moves seamlessly between an apprehension of the total active space and the amplification of its significant details. The movement is further emphasised by the viewer's freedom to move between the live ambient surround sound recording made in that space, and the focused commentaries of the glass studio artists which refer to specific processes and events that are unfolding.

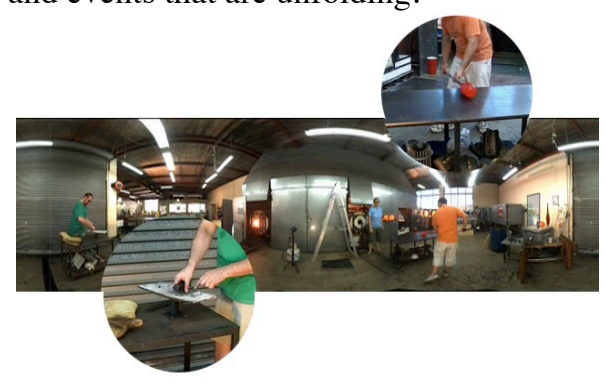

Figure 5. Diagram of close-up shots within the global recording 

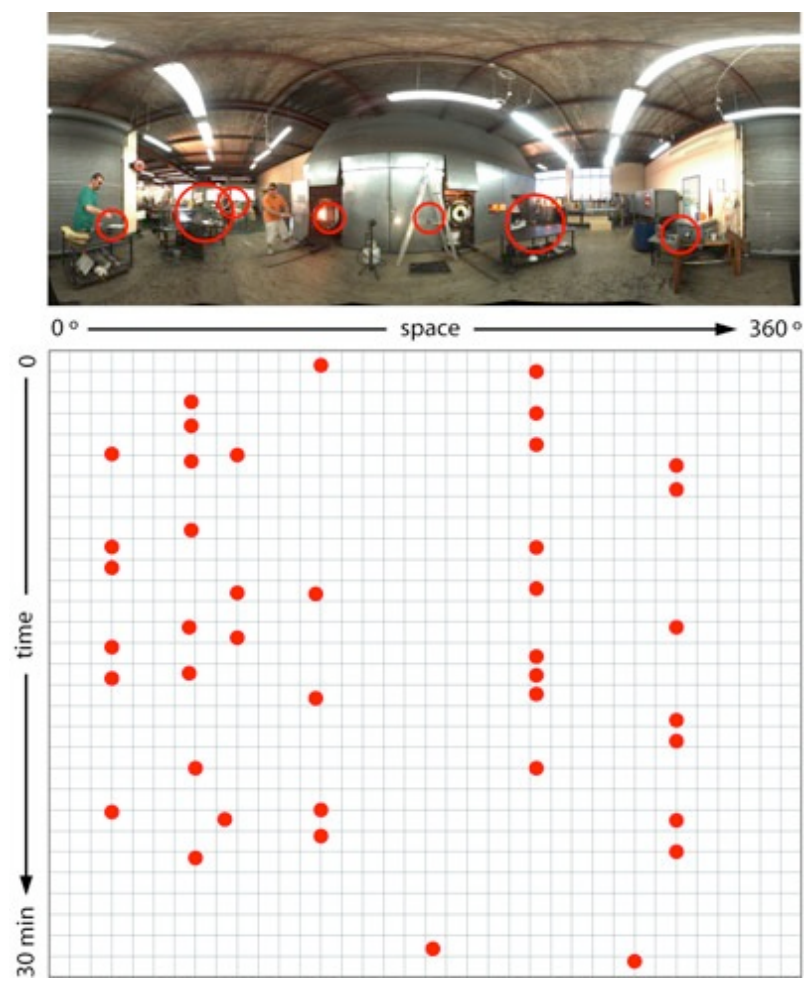

Figure 6. Diagram, Space-Time relation of augmented close-up shots in the $360^{\circ}$ global recording.

\subsubsection{Interaction}

The spherical movie is projected inside the dome with a coverage of 180 degree. A track ball integrated in the projector stand allows the user to rotate the projection freely while the movie is still running. Together with the image the multi-channel sound field is rotated accordingly. A simple vector based panning algorithm is used to distribute the multi-channel sound direction dependent on the user's principle point of view.

A control button activates a voice over commentary channel, whose content is related to the point of view and the time within the main spherical movie. This voice channel is mixed into the centre channel of the directional ambient audio. A second control button allows fast forward play back of the movie.

\subsubsection{Recording Technology}

The spherical video for Conversations@the Studio was shot with a Ladybug 2 camera system from Point Grey Research (1.2.2). The sound was recorded using a 4 channel Sound-Field microphone accompanied by a 5.1 Holophone. The individual directional sound channels are mapped to a point of view within the video and synchronised for playback in post production. 


\subsection{Historical Darling River Journey, 2009}

\subsubsection{Introduction}

A $360^{\circ}$ video recording on the Darling River in NSW Australia on the replica paddle steamer "PV Jandra" forms the background for this interactive video installation for the BackOBourke Exhibition Centre in Bourke NSW [5]. Additional narrative layers, superimposed historical photographs and a voice over channel by a local historian, provide information of the sights along the way. The visitors can freely control their gaze and jump to places of interest with a custom build user interface.
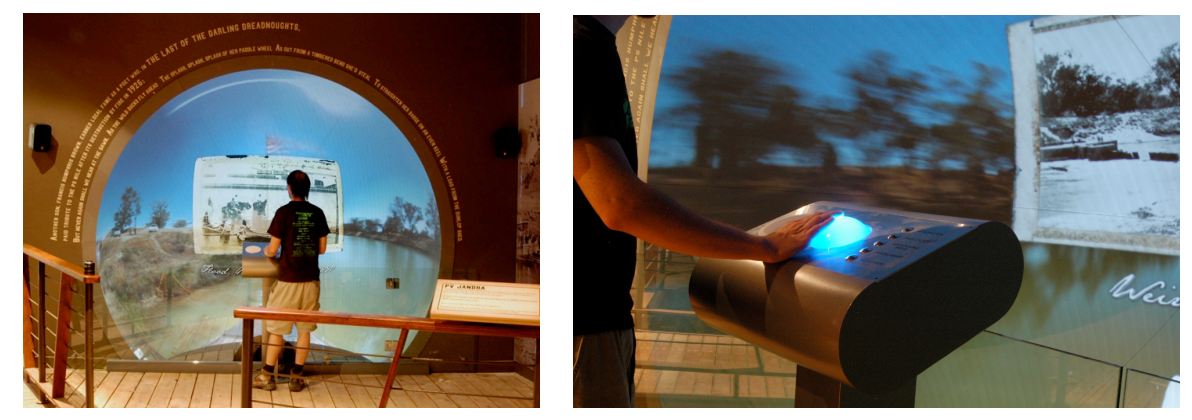

Figure 7. Installation at the BackOBourke Exhibition Centre, NSW Australia.

\subsubsection{Installation}

Similar to the Conversation@ the Studio application (2.1), this project is situated in the iDome environment, but uses a spherical mirror as reflection surface to fill the dome. Visitors are able to "look around" or translate the $180^{\circ}$ window of view within the $360^{\circ}$ global video recording by using a track ball interface. Together with the imagery, a four-channel ambient sound field turns.

Parallel and in time synchronicity to the 20 minute journey on the river, a second narrative layer comprised of historical photographs, maps and diagrams is augmented on the background of the global video recording. Together with a third layer, spoken voice commentary by a historian, these additional layers provide general background information and specific details about touristic sights along the river while the paddle steamer passes by. 


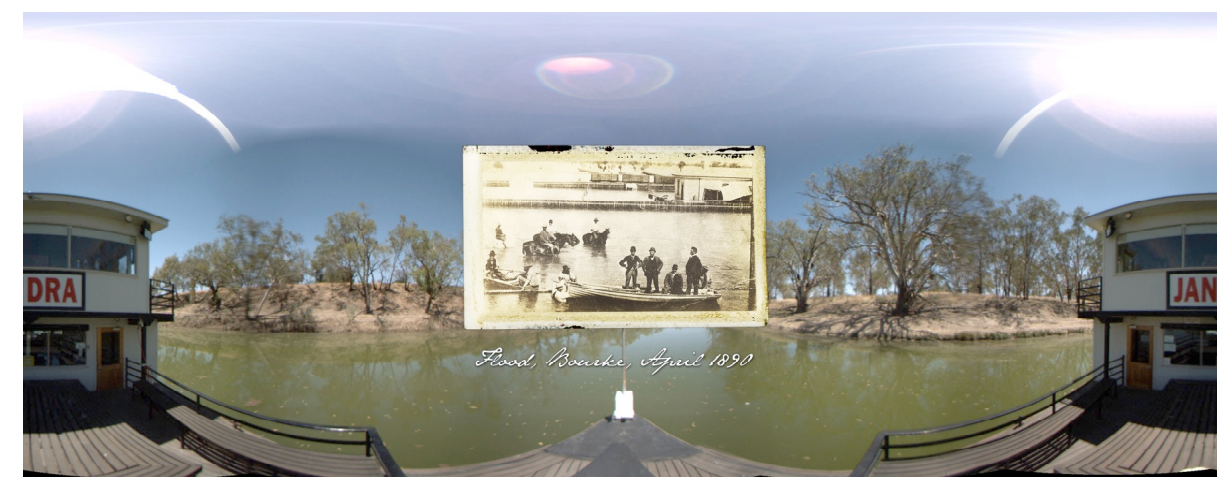

Figure 8. Frame from the global (Equirectangular projection, cropped) recording which forms the background layer in the application. Augmented is a historical photograph

\section{CONCLUSION}

Observation and feedback from visitors indicate that the outlined approach to Augmented Reality applications in a museum context constitutes an effective way to communicate with the general audience. The immersive quality of the display system and the novel use of global audio and video recording technologies attract visitors to engage with the system. The format the content is presented, the underlying narrative and an intuitive user interface keeps the visitor interested for long enough to get "the message" across.

The described framework of capture and display technologies together with multilayered narrative content presentation form a flexible system, which can be easily adapted for many applications in the educational and entertainment sector.

\section{ACKNOWLEDGEMENTS}

\section{Conversations @ the Studio, 2005}

Directors: Jeffrey Shaw, Dennis Del Favero, Neil Brown, Volker Kuchelmeister, Joachim Tesch, Nikos Papastergiadis, Scott McQuire, Andy Arthurs, Sarah Kenderdine, Kevin Sumption, Grace Cochrane

Glass Artists: Alexandra Chambers, Benjamin Edols, Kathy Elliott, Tom Rowney. Production and Technical Co-Ordinator: Volker Kuchelmeister. Lead Software Engineer: Joachim Tesch. Production Manager: Damian Leonard. Project Manager: Kate Dennis. Human Interface Designers: Volker Kuchelmeister, Jeffrey Shaw. Close-up Camera Operator: Greg Ferris. Lighting Designer: Max Harrison . Production Assistant: Sue Midgley. Spherical Video, Close-ups and Voice Commentaries: Volker Kuchelmeister. Sound Field Design: Andy Arthurs.

This project has been funded through an Australian Research Council Linkage Grant and an Australia Council Synapse Grant. The project has been co-produced by the UNSW iCinema Centre for Interactive Cinema Research, The University of 
Melbourne, Queensland University of Technology and the Powerhouse Museum, Sydney.

Darling River Journey, 2009

Produced at the UNSW iCinema Centre, Commissioned by Spinifex Interactive Sydney, Project Lead, Project Management, Concept, Cinematography: Volker Kuchelmeister, Software development: Ardrian Hardjono, Production management: Damian Leonard.

\section{REFERENCES}

1. Mike Bailey, Matt Clothier, Nick Gebbie. 2006. Realtime Dome Imaging and Interaction: Towards Immersive Design Environments. Proceedings of IDETC/CIE 2006

2. Paul Bourke. 2005. Spherical Mirror (Mirrordome): A New Approach To Hemispherical Dome Projection. Planetarian, Vol 34(4), December 2005, pp 6-9.

3. Paul Bourke. 2008. Low Cost Projection Environment for Immersive Gaming. JMM (Journal of MultiMedia), Volume 3, Issue 1, May 2008, pp 41-46.

4. Matthew Uyttendaele, Antonio Criminisi, Sing Bing Kang, Simon Winder, Richard Hartley, Richard Szeliski. 2003. High-quality Image-based Interactive Exploration of Real-World Environments. Technical Report. Microsoft Research October, 2003.

5. Historical Darling River Journey Project: Website and Video Documentation, UNSW $\begin{array}{lllll}\text { iCinema } & \text { Centre. } & \text { Retrieved } & 10 & \text { June }\end{array}$ (http://www.icinema.unsw.edu.au/projects/prj_bourke.html).

6. Conversations@the Studio Project: Website and Video Documentation Project, UNSW $\begin{array}{lllll}\text { iCinema } & \text { Centre. } & \text { Retrieved } & 10 & \text { June }\end{array}$ (http://www.icinema.unsw.edu.au/projects/prj_convostudio_1.html).

7. Spherecam: Website and Video Documentation, UNSW iCinema Centre. Retrieved 10 June 2009 (http://www.icinema.unsw.edu.au/projects/infra_spherecam_1.html).

8. iDome: Website and Video Documentation, UNSW iCinema Centre. Retrieved 10 June 2009 (http://www.icinema.unsw.edu.au/projects/infra dome.html).

9. Point Grey Research, Spherical Vision Products. Retrieved 10 June 2009 (http://www.ptgrey.com/products/spherical.asp). 